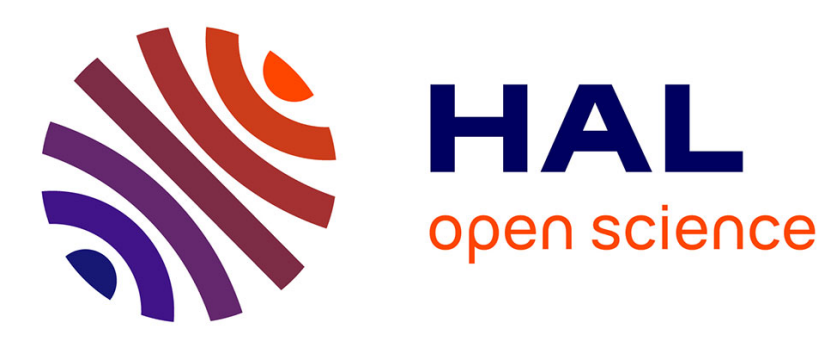

\title{
Force Tracking Impedance Control with Unknown Environment at the Microscale.
}

\author{
Bilal Komati, Cédric Clévy, Philippe Lutz
}

\section{To cite this version:}

Bilal Komati, Cédric Clévy, Philippe Lutz. Force Tracking Impedance Control with Unknown Environment at the Microscale.. IEEE International Conference on Robotics and Automation, ICRA'2014., Jan 2014, Hong Kong SAR China. pp.1-6. hal-01051650

\section{HAL Id: hal-01051650 \\ https://hal.science/hal-01051650}

Submitted on 25 Jul 2014

HAL is a multi-disciplinary open access archive for the deposit and dissemination of scientific research documents, whether they are published or not. The documents may come from teaching and research institutions in France or abroad, or from public or private research centers.
L'archive ouverte pluridisciplinaire HAL, est destinée au dépôt et à la diffusion de documents scientifiques de niveau recherche, publiés ou non, émanant des établissements d'enseignement et de recherche français ou étrangers, des laboratoires publics ou privés. 


\title{
Force Tracking Impedance Control with Unknown Environment at the Microscale
}

\author{
Bilal Komati, Cédric Clévy and Philippe Lutz
}

\begin{abstract}
A new method to estimate the environment parameters is proposed in order to perform force tracking in impedance control despite the presence of an unknown environment. In impedance force tracking, the location of the environment relative to the robot and the stiffness of the environment should be known. The proposed method estimates the environment location and stiffness using only force and position measurements. The study is done for microscale taking into consideration microscale specificities, especially pull-off force. The impedance control formulation is tested experimentally in a contact transition scenario consisting of a compliant microforce sensor mounted on a microrobotic positioner, and three compliant microstructures with different stiffness. A traditional double mass-spring-damper model of the overall robot is employed to develop the closed-loop impedance control.
\end{abstract}

\section{INTRODUCTION}

Force control is important for many microscale applications like biological, medical, microassembly, micromanipulation, etc. It improves the dexterity of the task by providing an additional feedback information. This feedback enables to detect any contact between the microrobot and the environment and guarantees the safety of both the microrobot and the environment by controlling the interaction forces[1]. Furthermore, at the microscale, surface forces are predominant and more influent than volume forces and induce some nonlinear effects, such as adhesion forces. It is notably manifested by pull-off forces, [2], which are naturally present and may affect any surface contact at the microscale. Microrobot control thus differ from macroscale control because microscale control has to overcome such microscale specificities. In addition, the integration of sensors is another limit in microscale which limits the automation and the development of some control algorithms. Although force control is important at the microscale, this topic is not covered like at the macroscale. Some microscale works use quasi-static models for hybrid force/position control [1], [3]. Others use explicit force control [4], [5], impedance control [4], [6] and vision-based force control [7]. In [4], a comparison between explicit force control and impedance control algorithms has been performed for microscale applications. It was proven experimentally that the impedance control presents better performances than explicit force control for microscale applications if the environment parameters are perfectly known which is not usually the case at the microscale. The importance of impedance control is that

The authors are with FEMTO-ST Institute, AS2M department, Université de Franche-Comté/CNRS/ENSMM, 24 rue Savary, 25000 Besançon, France, (e-mail: bilal.komati@femto-st.fr; cclevy@femto-st.fr; philippe.lutz@femto-st.fr). it enables dynamic control which improves the control of the microrobot relatively to the use of vision and static approaches at the microscale.

Previous works at the microscale approve that, similar to the macroscale, the impedance control technique proposed by Hogan [8] is a promising approach for controlling the dynamic interaction between a robot and an environment. However, one weak point of the impedance control is the lack of the direct force control capability by specifying a desired force which the explicit force control [9] and hybrid force/position control [10] do. Many researchers have paid attention to this fact and tried to solve the direct force control capability by using adaptive impedance control [11], [12], [13], [14], recursive least square [15], [16]. The recursive least square method used in [15] is difficult to implement and it estimates the environment mass, damping and stiffness within the framework of pure impedance control without tracking a desired force signal because the environment location is not estimated. Most of the developed works use adaptive impedance control to estimate the parameters of the environment. However, the implementation of the parameter estimation in the indirect adaptive controller requires data on the current position and velocity of the end-effector and the interaction force. In practice, especially at the microscale, accurate measurement of absolute velocity at the robot tip is difficult to achieve and induces a challenge in the application of the method. Furthermore, to use the algorithm, the user must specify the gain matrix of the adaptation law which increases the complexity of the method.

In this paper, a new method to estimate the environment location and stiffness is proposed in order to perform force tracking impedance control despite the presence of an unknown environment. This method requires only data on the current position of the end-effector and the interaction force and does not need to specify any gain. The complete control scheme with parameter estimation is easy to implement and guarantees force tracking. Furthermore, a new and easy method to deal with pull-off force is proposed in this paper.

The paper is organized as follows. The system model and impedance control strategy are developed in Section II. The new proposed method for the online estimation of the environment parameters is presented in Section III. Section IV shows the capability of force tracking despite the parameter estimation errors. The experimental setup used in this paper is presented in Section V. Section VI presents the experimental results obtained for the complete impedance control law with parameter estimation integrated into the experimental setup. Section VII concludes the article. 


\section{System Modeling And Control}

In this section, the model of the system used in this paper is discussed. Then, impedance control with force tracking strategy is presented.

\section{A. Model of the Robot and the Environment}

A classic robotic scenario is considered in this paper. Consider a robot end effector and let $f_{e}$ be the current contact force applied by the end effector to the environment once a contact between both is established. For modeling the robot/environment interaction, the environment is presented by a second order mass-spring-damper system like was done in [17]. The system model without contact and with contact is presented in Fig. 1, where $m_{e}, d_{e}$ and $k_{e}$ are respectively the mass, damping and stiffness of the environment, $m_{m}, d_{m}$ and $k_{m}$ are respectively the mass, damping and stiffness of the end effector manipulator, $x_{s}$ is the position of the positioning stage, $x_{s c}$ is the position of the positioning stage at contact, $x$ is the current position of the robot manipulator and $x_{e}$ is the position of the environment without any contact. Note that contrary to macrorobotics, most micropositioning devices are position controlled and not force controlled. In this paper, the position of the stage, $x_{s}$, is the command of the system and $f_{e}$ is the force to be controlled.

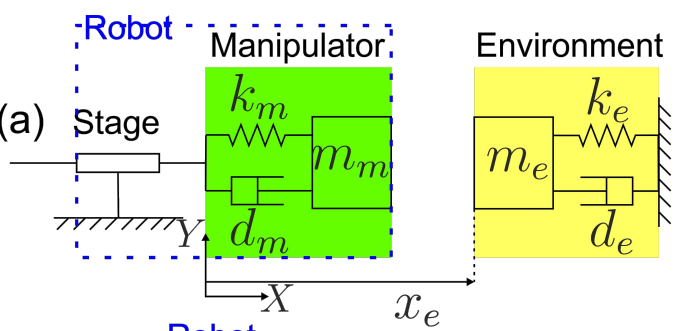

(b)

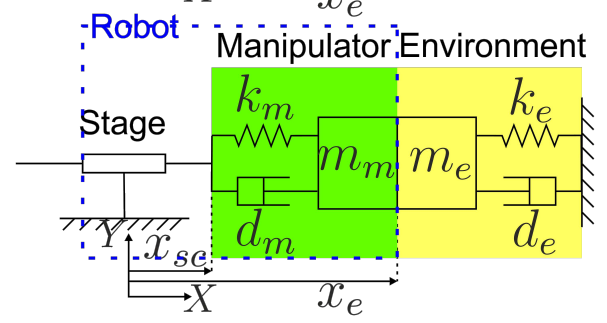

(c)

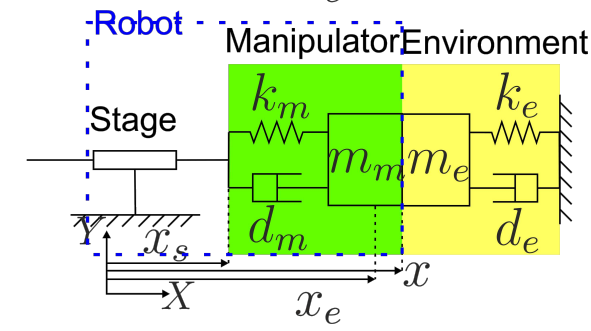

Fig. 1. System model based on mass spring damper: (a) without any contact between the end effector and the environment, (b) just at contact $f_{e}=0$ and (c) contact with $f_{e} \neq 0$.

Using Fig. 1-c, the force applied by the manipulator end effector to the environment is given by (1):

$$
f_{e}=F_{\text {end effector } \rightarrow \text { environment }}=m_{e} \ddot{x}+d_{e} \dot{x}+k_{e}\left(x-x_{e}\right)
$$

The force applied by the environment to the manipulator end effector is given by (2):

$$
f_{m}=-f_{e}=m_{m} \ddot{x}+d_{m}\left(\dot{x}-\dot{x}_{s}\right)+k_{m}\left(x-x_{s}\right)
$$

(2) could also be written like in (3)

$$
m_{m} \ddot{x}+d_{m} \dot{x}+k_{m} x+f_{e}=d_{m} \dot{x}_{s}+k_{m} x_{s}
$$

\section{B. Impedance Control with Force Tracking}

The objective of impedance control as proposed by Hogan in [8] is to establish a desired user-specified dynamical relationship, referred to as target impedance, between the end-effector position $x$ and the contact force $f_{e}$. Typically, the target impedance is chosen as a linear second order system, so that the dynamical relationship between the contact force $f_{e}$ and the end effector position $x$ can be controlled by a mass-spring-damper system. A common formulation of the target impedance is given by (4) where $M_{d}, D_{d}$ and $K_{d}$ are respectively the desired mass, damping and stiffness of the target impedance, $x_{r}$ is the reference position, $f_{r}$ is the reference force and $e_{f}$ is the force error. The classical impedance control scheme is presented in Bloc I of Figure 2.

$$
M_{d}\left(\ddot{x}-\ddot{x}_{r}\right)+D_{d}\left(\dot{x}-\dot{x}_{r}\right)+K_{d}\left(x-x_{r}\right)=f_{r}-f_{e}=e_{f}
$$

In any practical implementation of impedance control, a position tracking error, due to the dynamics of the robot, will appear between the end effector position and the desired position calculated by (4). In this case, (4) could be written as in (5) where $x_{d}$ is desired position trajectory generated.

$$
M_{d}\left(\ddot{x}_{d}-\ddot{x}_{r}\right)+D_{d}\left(\dot{x}_{d}-\dot{x}_{r}\right)+K_{d}\left(x_{d}-x_{r}\right)=f_{r}-f_{e}
$$

Thus, an inner loop Position Control Law (PCL) should be used to let $x$ track $x_{d}$. Let $\varepsilon=x_{d}-x$ represents the position error between the desired position generated by (5) and the current position of environment. Using the result of [11], the steady state force error is given by:

$$
e_{f}^{s s}=k_{e q}\left[\frac{f_{r}}{k_{e}}+x_{e}+\varepsilon-x_{r}\right]
$$

where $k_{e q}=\frac{K_{d} k_{e}}{K_{d}+k_{e}}$ is the equivalent stiffness of the target impedance and the environment. Equation (6) shows that if an inner loop position control law is used in such a way to have a zero steady state position error $(\varepsilon \rightarrow 0)$ and the reference position is chosen precisely as in (7), then the steady state force error will be cancelled $\left(e_{f}^{s s} \rightarrow 0\right)$.

$$
x_{r}=x_{e}+\frac{f_{r}}{k_{e}}
$$

Equation (7) shows that if the precise location of the environment $x_{e}$ and the exact value of the environment stiffness $k_{e}$ are known then a reference position trajectory $x_{r}$ could be generated according to (7) to exert the desired contact force $f_{r}$ on the environment. However, in practice the values $x_{e}$ and $k_{e}$ are not known perfectly and, as a result, the desired force 
$f_{r}$ will not be exerted on the environment. Let $\left(\Delta x_{e}, \Delta k_{e}\right)$ be the uncertainties on the location of the environment, that is:

$$
\begin{aligned}
& x_{e}=\hat{x}_{e}+\Delta x_{e} \\
& k_{e}=\hat{k}_{e}+\Delta k_{e}
\end{aligned}
$$

where $\hat{x}_{e}$ and $\hat{k}_{e}$ are respectively the estimated values of $x_{e}$ and $k_{e}$. Then using the reference position $x_{r}=\hat{x}_{e}+\frac{f_{r}}{\hat{k}_{e}}$ given in (7), the steady state force error given in (6) could be written as in (9) which shows that if the $\left(\Delta x_{e}, \Delta k_{e}\right)$ are big then the steady state force error is also big.

$$
e_{f}^{s s}=\frac{K_{d}}{K_{d}+\hat{k}_{e}+\Delta k_{e}}\left[\hat{k}_{e} \Delta x_{e}-\frac{\Delta k_{e}}{\hat{k}_{e}} f_{r}+\left(\Delta x_{e}\right)\left(\Delta k_{e}\right)\right]
$$

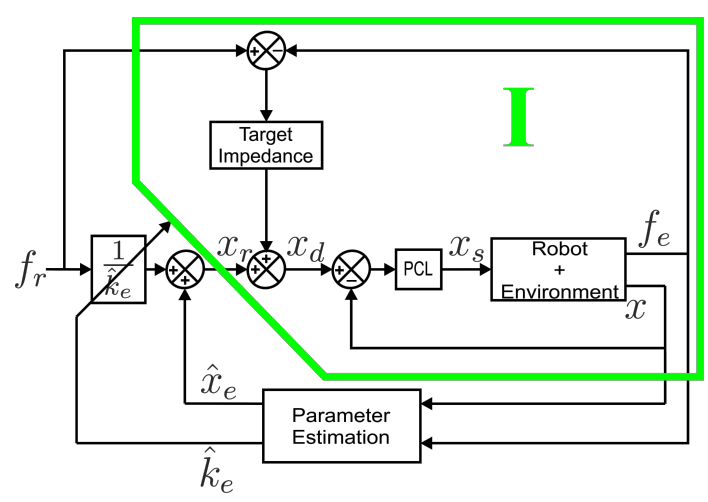

Fig. 2. Position based impedance control system with force tracking where PCL is an inner loop Position Control Loop. Bloc I is the classical position based impedance control scheme without force tracking.

\section{ENVIRONMENTAL LOCATION AND STIFFNESS ESTIMATION TECHNIQUE}

As shown in the previous section, in order to achieve a force tracking in the impedance control scheme, the environment location $x_{e}$ and the environment stiffness $k_{e}$ should be known or estimated. Many techniques have been used in the past including adaptive control and recursive least square. These methods are usually difficult to be implemented to the system and require data on the current position and velocity of the end effector and the interaction forces which is difficult at the microscale due to the lack of velocity sensors. In this section, a new simple on line estimation method is proposed in order to estimate the environment location $x_{e}$ and its stiffness $k_{e}$. The proposed method is easy to be implemented, it does not need to specify adaptive gains and it requires only data on the location of the end-effector and the interaction forces.

The first step is to determine the location of the environment $x_{e}$. The idea is to apply the impedance control law presented in the previous section using assumptions $\hat{x}_{e}^{0}$ and $\hat{k}_{e}^{0}$ of $x_{e}$ and $k_{e}$. Once the control law is applied, a transition between non contact and contact happens as shown in Fig. 1-a and 1-c. During this transition, an on line acquisition of the force and position measurements is achieved and when a contact is detected the location of the environment, $\hat{x}_{e}$, is determined by the past value of the position measurement.
Due to the noisy force signal measurement (amplitude of noise $\pm 5 \mu \mathrm{N}$ in our case), a dead zone is defined $(5 \mu \mathrm{N})$ and a contact is taken into consideration if the force measurement is bigger than the dead zone.

Once the environment location is estimated, the environment stiffness is estimated using the static part of (1):

$$
\hat{k}_{e}=\frac{f_{e}}{x-\hat{x}_{e}} \quad \text { if } x>\hat{x}_{e}
$$

Note that ignoring the dynamical part of (1) could modify the desired dynamic of the system but it will not affect the steady state part. Using (6), we can write:

$$
x_{r}=\hat{x}_{e}+\frac{f_{r}}{\hat{k}_{e}}=\hat{x}_{e}+\frac{f_{r}}{f_{e}}\left(x-\hat{x}_{e}\right) \Leftrightarrow x_{r}-\hat{x}_{e}=\frac{f_{r}}{f_{e}}\left(x-\hat{x}_{e}\right)
$$

Using (11) if $x$ tracks $x_{r}$, then $f_{e}$ will track $f_{r}$.

The complete impedance control scheme with parameter estimation used in this paper is given in Fig. 2.

\section{FORCE TRACKING DESPITE ESTIMATION ERRORS}

Using (1) and (10), the steady state force applied on the environment, $f_{e}^{s s}$, could be written in the two forms of (12):

$$
f_{e}^{s s}=\hat{k}_{e}\left(x^{s s}-\hat{x}_{e}\right)=k_{e}\left(x^{s s}-x_{e}\right)
$$

where $x^{s s}$ is the position of the environment in steady state. Replacing (8) in (12), the following could be deduced:

$$
\hat{k}_{e} x^{s s}-\hat{k}_{e} \hat{x}_{e}=\left(\hat{k}_{e}+\Delta k_{e}\right)\left(x^{s s}-\hat{x}_{e}-\Delta x_{e}\right)
$$

After developing (13), (14) can be derived:

$$
\Delta k_{e}=\frac{\hat{k}_{e} \Delta x_{e}}{x^{s s}-\hat{x}_{e}-\Delta x_{e}}
$$

Using (12), $x^{s s}-\hat{x}_{e}=\frac{f_{e}^{s s}}{\hat{k}_{e}}$, and replacing the latter in (14), (15) can be derived:

$$
\Delta k_{e}=\frac{\hat{k}_{e}^{2} \Delta x_{e}}{f_{e}^{s s}-\hat{k}_{e} \Delta x_{e}}
$$

Thus, the error in the estimation of the stiffness of the environment, $\Delta k_{e}$, increases if the error of the estimation of the position, $\Delta x_{e}$, increases as (15) shows. If the estimation of the position of the environment is small then the estimation of the stiffness is small.

Replacing (15) in (9), (16) can be derived:

$$
e_{f}^{s s}=\frac{-K_{d} \hat{k}_{e} \Delta x_{e}}{K_{d} f_{e}^{s s}+\hat{k}_{e} f_{e}^{s s}-K_{d} \hat{k}_{e} \Delta x_{e}} e_{f}^{s s}
$$

Equation (16) is true if and only if:

$$
\left\{\begin{array}{l}
e_{f}^{s s}=0 \\
\text { or: } \\
\frac{-K_{d} \hat{k}_{e} \Delta x_{e}}{K_{d} f_{e}^{s s}+\hat{k}_{e} f_{e}^{s s}-K_{d} \hat{k}_{e} \Delta x_{e}}=1
\end{array}\right.
$$

Equation (17) is equivalent to:

$$
\left\{\begin{array}{l}
e_{f}^{s s}=0 \\
\text { or: } \\
f_{e}^{s s}=0
\end{array}\right.
$$


Equation (18) shows that using the parameter estimation technique presented in section III, once a contact force is detected $\left(f_{e} \neq 0\right)$, the steady state force error is always zero and the force tracking is guaranteed even if big estimation errors exist on $x_{e}$ and $k_{e}$ because the errors are compensated.

\section{EXPERIMENTAL SETUP}

The hardware shown in Fig. 3 was used to control the interaction between a MEMS force sensor and a compliant environment. A microstructure made of $300 \mu \mathrm{m}$ of thickness of glass was used as the passive compliant mechanism of the environment and it was attached to a stationary base. A Femto-Tools force sensing probe FT-S270, with a sensing range of $2 \mathrm{mN}$ and a resolution of $0.4 \mu \mathrm{N}$, used as an end effector, was attached to a positioning stage. The force sensor comprises a probe of $3 \mathrm{~mm}$ of length and $50 \mu \mathrm{m}$ of thickness, that moves along $X$ direction (according to Fig. 3) once a force is applied at its tip. The displacement is converted into a voltage thanks to a capacitive variation measured by a dedicated circuit. The maximal contact surface between the force sensor and the environment is $50 \mu \mathrm{m} \times 50 \mu \mathrm{m}$. The positioning stage is a PXY D12 - piezo XY scan positioner from PiezoSystemJena with a travel range of $200 \mu \mathrm{m}$. It has an internal capacitive sensor to measure the displacement of the stage along its two axis. The positioning stage was controlled and the force and the position feedback were acquired through a dSPACE1104 board.

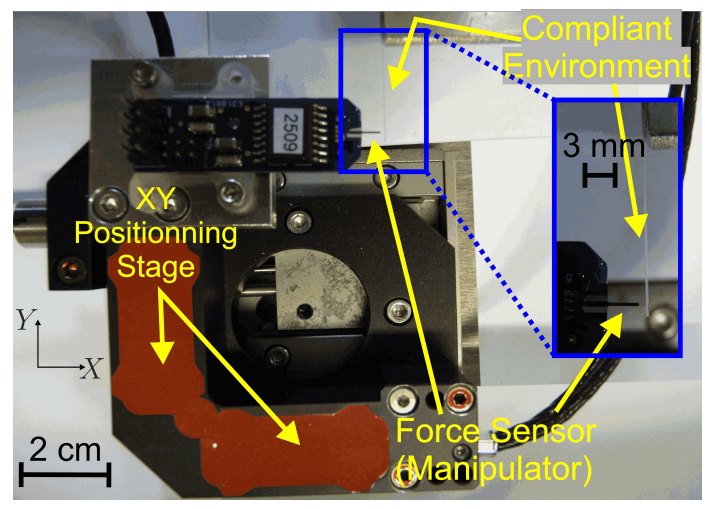

Fig. 3. Experimental setup.

\section{EXPERIMENTAL RESULTS}

In this section, the impedance control with force tracking scheme presented in Section II is implemented via a dSPACE 1104 board, with a sampling frequency $10 \mathrm{kHz}$ without any knowledge of the location and the stiffness of the environment. The method presented in Section III is used to estimate $x_{e}$ and $k_{e}$. First, the maximal errors on the estimation of the environment parameters $\left(x_{e}\right.$ and $k_{e}$ ) are evaluated using the experimental setup presented in section $\mathrm{V}$. Then, several object stiffness are used to test the effectiveness of the impedance control with force tracking scheme presented in Section II.

\section{A. Maximal error on the estimation of $x_{e}$}

The maximal speed of the positioning stage is $1 \mathrm{~mm} / \mathrm{s}$. The sampling time used in this paper is $100 \mu \mathrm{s}$. Indeed, the maximal displacement of the positioning stage in $100 \mu \mathrm{s}$ is $100 \mathrm{~nm}$. Using these results, the maximal error on the estimation of the environment location is $100 \mathrm{~nm}$.

\section{B. Maximal error on the estimation of $k_{e}$}

The error on the estimation of the stiffness could be deduced from (15) which can be written as:

$$
\frac{\Delta k_{e}}{\hat{k}_{e}}=\frac{\hat{k}_{e} \Delta x_{e}}{f_{e}^{s s}-\hat{k}_{e} \Delta x_{e}}
$$

Inducing the maximal error on the estimation of the location $(0.1 \mu \mathrm{m})$ in $(19)$, the following can be deduced:

$$
\hat{k}_{e} \Delta x_{e}<0.1 \hat{k}_{e}
$$

Using (19) and (20), the following is deduced:

$$
\frac{\Delta k_{e}}{\hat{k}_{e}}<\frac{0.1 \hat{k}_{e}}{f_{e}^{s s}-0.1 \hat{k}_{e}}
$$

Equation (21) shows that the relative error on the estimation of the stiffness increases if the stiffness is big and the steady state force is small. Considering $f_{e}^{s s}=1000 \mu \mathrm{N}$ and the stiffness of the environment is $1000 \mathrm{~N} / \mathrm{m}$, which is a big stiffness for the microcomponents, then, using (21) the maximal error on the estimation of the stiffness could reach $11 \%$. The calculated errors on the location and the stiffness of the environment are considered in the worst case and in experiments they are much smaller. However, as already said, the parameter estimation errors are compensated to have finally a zero steady state force error as shown in section IV.

\section{Experimental investigations}

To test the impedance control with force tracking scheme presented in Section II, several object stiffness are used. Initial values $\hat{x}_{e}^{0}$ and $\hat{k}_{e}^{0}$ are used as assumptions for $x_{e}$ and $k_{e}$. Two scenarios exist in function of the presumption of the location of the environment $\hat{x}_{e}^{0}$ :

- if $x_{r}=\hat{x}_{e}^{0}+\frac{f_{r}}{\hat{k}_{e}^{0}}<x_{e}$ : in this case, no contact appears and the parameters could be estimated and accordingly the force reference could not be tracked like it was shown in the second equation of (18) $\left(f_{e}^{s s}=0\right)$,

- if $x_{r}=\hat{x}_{e}^{0}+\frac{f_{r}}{\hat{k}_{e}^{0}}>x_{e}$ : in this case, a contact appears and the parameters are estimated and the force reference is tracked like it was shown in the first equation of (18) $\left(e_{f}^{s s}=0\right)$.

Taking into consideration the two scenarios, the assumption of the environment location $\hat{x}_{e}^{0}$ is chosen big enough in order to guarantee that a contact happens without any force reference. The location is then estimated to be $\hat{x}_{e}$ and the end effector's position $x$ tracks $\hat{x}_{e}$. Once a reference force $f_{r}$ is applied, the controller manages to let $f_{e}$ tracks $f_{r}$.

Figure 4 shows the impedance control scheme with force tracking in presence of an environment with a stiffness of $72.5 \mathrm{~N} / \mathrm{m}$. The initial position of the environment is 12.5 
$\mu \mathrm{m}$. At the beginning of the experiment, the controller is turned off, the assumptions of the position and the stiffness of the environment are respectively $30 \mu \mathrm{m}$ and $100 \mathrm{~N} / \mathrm{m}$. At $t=0.04 \mathrm{~s}$; the controller is turned $O N$ and the stage moves to let $x_{r}$ tracks $\hat{x}_{e}^{0}$ because the force reference $f_{r}$ and the measured force $f_{e}$ are null. As $\hat{x}_{e}^{0}>x_{e}$, a contact appears at $t=0.05 \mathrm{~s}$. Because a force is detected, $\hat{x}_{e}$ is estimated and it is equal to $12.51 \mu \mathrm{m}$. The estimation of the environment location is fixed in the rest of the experiment. A reference force is set to $1 \mathrm{mN}$ at $t=0.2 \mathrm{~s}$. The estimation of the stiffness along (10) starts and the controller is able to track the force reference with a response time of $150 \mathrm{~ms}$ and an overshoot of $20 \%$. The stiffness of the environment is estimated to be around $71.95 \mathrm{~N} / \mathrm{m}$. The estimation of the stiffness will then be used in the rest of the experiment. To test the capability of the controller and the effectiveness of the parameter estimation technique, another force reference of $1.5 \mathrm{mN}$ is applied at $t=0.666 \mathrm{~ms}$. The controller is able to cancel the steady state force error in $70 \mathrm{~ms}$ with no overshoot. The controller is tested in the other direction i.e. if the force reference decreases. The controller performances are almost the same if the force reference goes from $1.5 \mathrm{mN}$ to $1 \mathrm{mN}$ and from $1 \mathrm{mN}$ to zero. The error on the estimation of the environment is $\Delta x_{e}=0.01 \mu \mathrm{m}$ and on the environment stiffness is $\Delta k_{e}=0.55 \mathrm{~N} / \mathrm{m}$. However, despite the error on the parameter estimation, $f_{e}$ tracks $f_{r}$. At $t=1.885 \mathrm{~s}, f_{e}$ returns to zero but the contact is not broken due to the pull-off force which has sticking effects and prevent the breaking of the contact. A simple method is proposed to break the contact by cancelling the effect of the pull-off force by applying a negative force reference as shown in Figure 5.
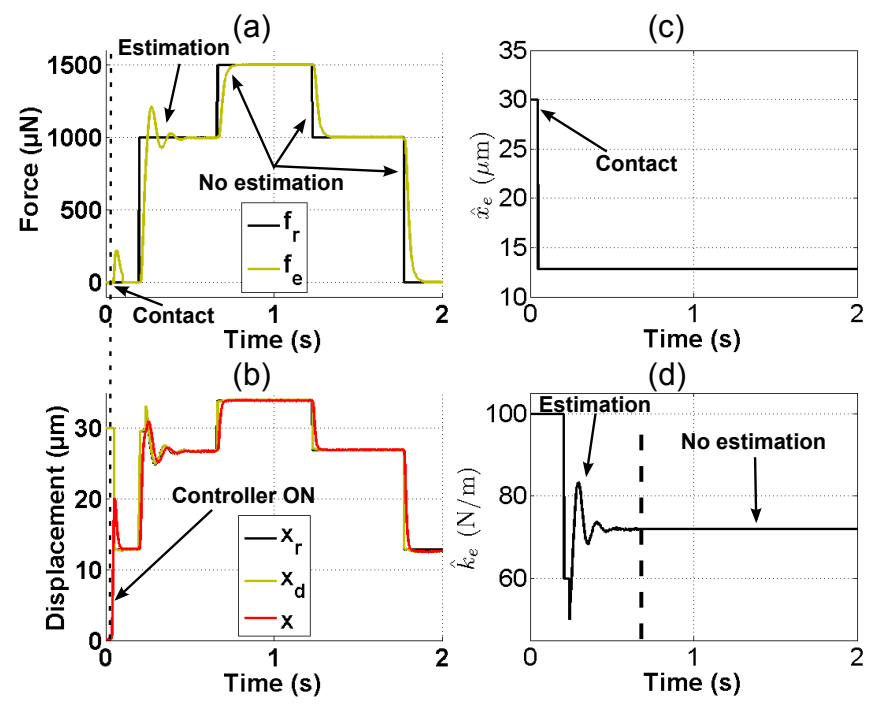

(d)

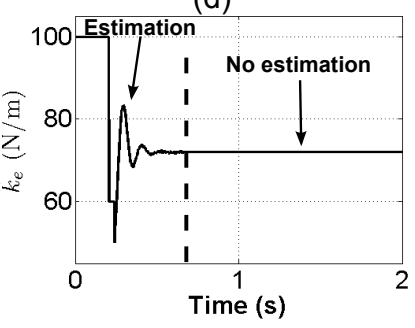

Fig. 4. Impedance control with force tracking in presence of an environment of stiffness $72.6 \mathrm{~N} / \mathrm{m}$ (a) contact force $f_{e}$ is compared relative to the force reference $f_{r}$ (b) position of the environment is compared to the reference $x_{r}$ and the desired $x_{d}$ positions (c) Environment location $\hat{x}_{e}$ (d) Environment stiffness $\hat{k}_{e}$.

Figure 5 shows the same procedure used in Figure 4 in presence of an environment with stiffness $976 \mathrm{~N} / \mathrm{m}$ which is a relative stiff environment in microscale applications. The en- vironment location is first estimated with an error of $0.05 \mu \mathrm{m}$. Once a force reference is applied, some oscillations appear in the response of the system. Despite the big overshoot present in the system, the stiffness of the environment is estimated $930 \mathrm{~N} / \mathrm{m}$ with a small error $(5 \%)$ and the force $f_{e}$ tracks the reference force $f_{r}$ with a response time of $386 \mathrm{~ms}$ and an overshoot of $65 \%$.

As already discussed, in order to break the contact, a negative force reference is applied at $t=1.321 \mathrm{~s}$ and a pull-off force of $116 \mu \mathrm{N}$ appears at $t=1.342 \mathrm{~s}$ as shown in Figure 5. Then, the contact is broken and the force $f_{e}$ returns to zero. Hence, another advantage of the proposed control method is that it can deal with pull-off force by simply applying negative force reference. (a)

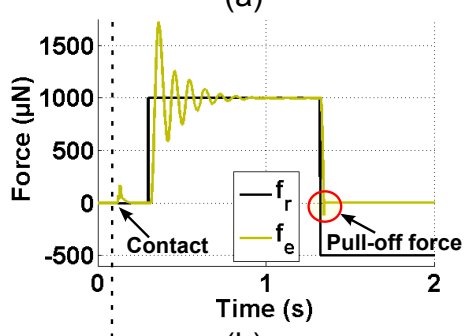

(b)

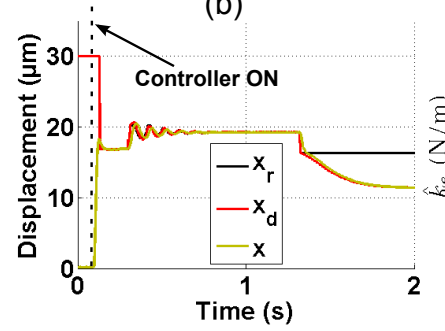

(c)

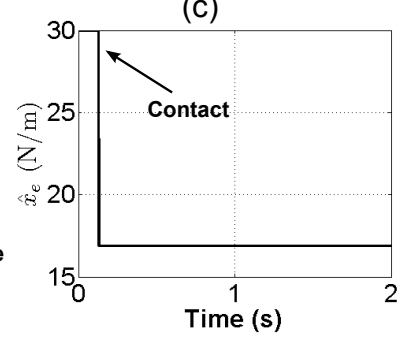

(d)

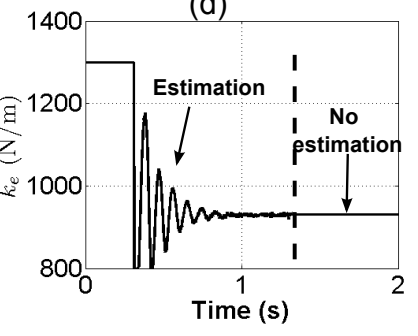

Fig. 5. Impedance control with force tracking in presence of an environment of stiffness $976 \mathrm{~N} / \mathrm{m}$ (a) contact force $f_{e}$ is compared relative to the force reference $f_{r}$ (b) position of the environment is compared to the reference $x_{r}$ and the desired $x_{d}$ positions (c) Environment location $\hat{x}_{e}$ (d) Environment stiffness $\hat{k}_{e}$. A pull-off force appears at $t=1.342 \mathrm{~s}$

In Table I, a comparison between the behavior of the proposed control scheme in presence of three environments with three different stiffness is done. The steady state force error is null in the three cases despite the parameter estimation errors. The overshoot of the system and the response time increase if the stiffness of the environment increases. The estimation of the parameters is precise with a relative small error in the three cases which is not only important but so difficult in microscale applications. The force tracking impedance control scheme with the proposed estimation method presents better performances comparing it to other methods of parameter estimation where bigger force error exists like in [6]. Indeed, the response time is faster than in [12], [13], [16] and the overshoot is smaller than in [11], [13], [16]. The maximal estimation error of the environment stiffness using the proposed method is $11 \%$ where it is $12.5 \%$ using the indirect adaptive control [16]. 


\begin{tabular}{|c|c|c|c|}
\hline Stiffness(N/m) & 72.5 & 469 & 976 \\
\hline$e_{f}^{s s}$ & 0 & 0 & 0 \\
\hline$\Delta x_{e}(\mu \mathrm{m})$ & 0.1 & 0.087 & 0.05 \\
\hline$\Delta k_{e}(\%)$ & 0.7 & 3.2 & 5 \\
\hline Response time $(\mathrm{ms})$ & 150 & 192 & 386 \\
\hline Overshoot $(\%)$ & 20 & 25 & 65 \\
\hline
\end{tabular}

TABLE I

EXPERIMENTAL RESULTS COMPARISON FOR THREE ENVIRONMENTS WITH THREE DIFFERENT STIFFNESSES.

\section{CONCLUSION}

In this paper, a new method to estimate the environment parameters is proposed in order to perform force tracking in impedance control despite the presence of an unknown environment. The proposed method estimates the environment location and stiffness using only force and position measurements. The study is done for microscale taking into consideration microscale specificities, especially pull-off force.

In impedance force tracking, the location of the environment relative to the robot and the stiffness of the environment should be known which is difficult, especially at the microscale. The proposed estimation method is able to estimate the environment location with an error less than $0.1 \mu \mathrm{m}$ and the environment stiffness less than $11 \%$. These values are considered in the worst cases while in experiments the environment stiffness error was smaller than $5 \%$. However, the control scheme is able to cancel the steady state force error despite the estimation errors on the environment parameters. The complete impedance control scheme is able to track the force reference with a response time of $150 \mathrm{~ms}$ and an avershoot of $20 \%$ in presence of flexible environments $\left(k_{e}<100 \mathrm{~N} / \mathrm{m}\right)$ while the reponse time reaches $386 \mathrm{~ms}$ and the overshoot reaches $65 \%$ in presence of stiff environment (around 1000N/m). Furthermore, a simple method to deal with pull-off force is proposed by applying a negative force reference to the system.

Three main advantages exist for using the proposed estimation method relative to other existing methods. The first advantage is that it requires data only on the current location of the end-effector and the interaction force which is an advantage comparing to the indirect adaptive impedance control where an additional data on the velocity of the endeffector is required. This point is important, especially at the microscale, because accurate measurement of absolute velocity at the robot tip is difficult to achieve and most of the microscale sensors measures only the position of the endeffector which induces a challenge in the application of the method. The second advantage is that no adaptive gains need to be specified in order to perform parameter estimation which simplifies the use of the estimation method. The third advantage is that this method is easy to be implemented and presents better performances than existing work on adaptive impedance control. Hence, the complete force tracking impedance control scheme with the proposed parameter estimation method is suitable for microscale applications where the stiffness and the location of the environment are unknown and variable.

This paper shows that the use of impedance control with force tracking for microscale applications is a promising topic for force control of some microrobotics, robotic microassembly and micromanipulation applications. This work was done for a 1 DOF moving stage case study and can be performed for more complex systems.

\section{ACKNOWLEDGMENT}

These works have been partially funded by the FrancheComté region and the Labex ACTION project (contract "ANR-11-LABX-01-01").

\section{REFERENCES}

[1] B. Komati, K. Rabenorosoa, C. Clévy, and P. Lutz, "Automated guiding task of a flexible micropart using a two-sensing-finger microgripper," IEEE Trans. on Automation, Science and Engineering, vol. 10 , no. 3, pp. 515-524, July 2013.

[2] K. Rabenorosoa, C. Clévy, P. Lutz, M. Gauthier, and P. Rougeot, "Measurement of pull-off force for planar contact at the microscale," Micro Nano Letters, vol. 4, pp. 148-154, 2009.

[3] K. Rabenorosoa, C. Clévy, and P. Lutz, "Hybrid force/position control applied to automated guiding tasks at the microscale," IEEE/RSJ Int. Conf. on Intelligent Robots and Systems, pp. 4366-4371, Oct. 2010.

[4] B. Komati, M. Pac, I. Ranatunga, C. Clévy, D. Popa, and P. Lutz, "Explicit force control v.s. impedance control for micromanipulation," ASME International Design Engineering Technical Conferences \& Computers and Information in Engineering Conference (IDETC), Portland, USA, August 2013.

[5] M. Rakotondrabe and A. Ivan, "Development and force/position control of a new hybrid thermo-piezoelectric microgripper dedicated to micromanipulation tasks," IEEE Trans. on Automation Science and Engineering, vol. 8, no. 4, p. 824834, 0ct. 2011.

[6] Q. Xu, "Precision position/force interaction control of a piezoelectric multimorph microgripper for microassembly," IEEE Trans. on Automation, Science and Engineering, vol. 10, no. 3, pp. 503-514, July 2013.

[7] J. Wason, J. Wen, J. Gorman, and N. Dagalakis, "Automated multiprobe microassembly using vision feedback," IEEE Trans. on Robotics, vol. 28, no. 5, pp. 1090-1103, Oct. 2012.

[8] N. Hogan, "Impedance control - an approach to manipulation. i- theory. ii- implementation. iii- applications," ASME Trans. J. of Dynamic Systems and Measurement Control B, vol. 107, pp. 1-24, 1985.

[9] R. Volpe and P. Khosla, "A theoretical and experimental investigation of explicit force control strategies for manipulators," IEEE Trans. on Automatic Control, vol. 38, no. 11, pp. 1634-1650, Nov. 1993.

[10] M. Raibert and J. J. Craig, "Hybrid position/force control of manipulators," Trans. of ASME, Journal of Dynamic Systems, Measurement, and Control, vol. 102, pp. 126-133, 1981.

[11] H. Seraji and R. Colbaugh, "Force tracking in impedance control," Int. J. of Robotics Research, vol. 16, no. 1, pp. 97-117, Feb. 1997.

[12] S. Singh and D. Popa, "An analysis of some fundamental problems in adaptive control of force and impedance behavior: theory and experiments," IEEE Trans. on Robotics and Automation, vol. 11, no. 6, pp. 912-921, 1995.

[13] S. Misra and A. Okamura, "Environment parameter estimation during bilateral telemanipulation," Symp. on Haptic Interfaces for Virtual Environment and Teleoperator Systems, pp. 301-307, March 2006.

[14] W. Xu, C. Cai, M. Yin, and Y. Zou, "Time-varying force tracking in impedance control," IEEE Conf. on Decision and Control (CDC), pp. 344-349, 2012.

[15] L. Love and W. Book, "Environment estimation for enhanced impedance control," IEEE Int. Conf. on Robotics and Automation, vol. 2, pp. 1854-1859, 1995.

[16] D. Erickson, M. Weber, and I. Sharf, "Contact stiffness and damping estimation for robotic systems," Int. J. of Robotics Research, vol. 22, no. 1, pp. 41-57, Jan 2003.

[17] H. Kazerooni, T. Sheridan, and P. Houpt, "Robust compliant motion for manipulators, parts i and ii," IEEE J. of Robotics and Automation, vol. 2, no. 2, pp. 83-105, June 1986. 